\title{
Oral Microbiome Homeostasis: The New Frontier in Oral Care Therapies
}

\author{
Gerald P. Curatola* \\ NYU College of Dentistry, New York, USA
}

Received: December 18, 2013; Accepted: December 19, 2013; Published: December 22, 2013

"Corresponding author: Gerald P. Curatola, D.D.S., New York, USA, E-mail: gcuratola@nyu.edu

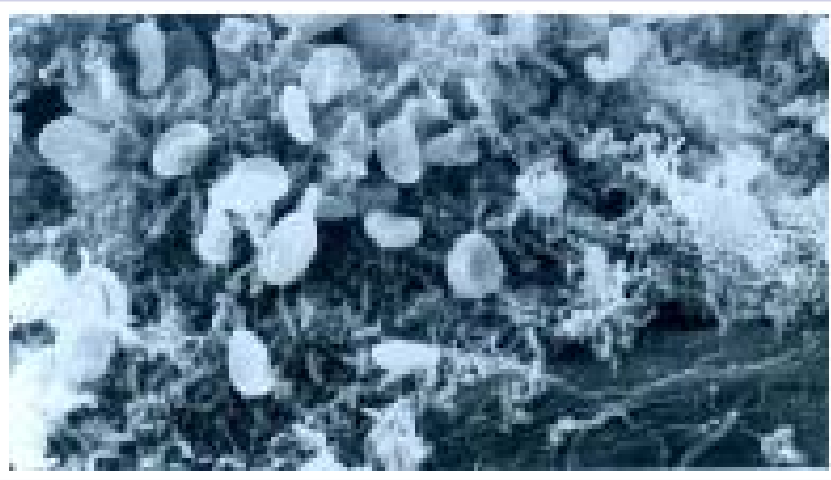

Emerging science of the Human Microbiome is radically changing our understanding and approach toward oral health, systemic immunity and total body wellness.

The 2000 Report on Oral Health by the U.S. Surgeon General declared that oral health reflects general health and it is essential to achieve a state of wellness. Conversely, it went on to report that oral diseases and disorders, in and of themselves, negatively impact health and wellbeing throughout life, and more scientific research remains critical to reducing the impact from the burden of oral diseases throughout the body [1]. That time has come. In the last decade, virtually everything we thought we knew about the origins and causes of oral disease has been turned upside down. The dental research community learns more every day about the unique and fascinating makeup of the oral microbiome, the unique natural ecology of the mouth, and the vital role this plays in oral and overall health. The term microbiome was coined by Joshua Lederberg "to signify the ecological community of commensal, symbiotic, and pathogenic microorganisms that literally share our body space and have been all but ignored as determinants of health and disease" [2]. The human mouth is the residence of one of the most diverse microbiomes in the entire human body comprised of over 600 prevalent taxa at the species level, with distinct subsets predominating in different habitats, and an estimated 20 billion resident microbes existing in the oral cavity at any one time [3].

Month after month, new research is published that expands what we know about the tiny resident organisms living in the mouth's microbiome, and sheds more light on how bacteria and other microorganisms may be implicated in non-oral health outcomes such as heart disease, stroke[4], or complications with pregnancy [5]. Microbiome communities occur naturally throughout nature and the body from thin wet spaces between layers of rock, to our hair, skin, eyes, entire digestive tract, lungs and urogenital system. The number of these organisms in these communities in and on our bodies is believed to be $10 \mathrm{x}$ greater than our own human cells and the genetic information they possess being 100x greater. This new understanding of the essential synergistic and symbiotic relationship that exists between man and microbe has been the focus of the Human Microbiome Project supported by the National Institute of Health. It has also redefined our "humanity" to be that of a microbiome/ human "superorganism" [6].

For decades, dental professionals have subscribed to an oral healthcare approach that holds the necessity to disinfect or even sterilize the mouth at its foundation. This "scorched earth policy" stems from research first conducted by Robert Koch in the late 1800 's. At that time, Koch began to assign specific bacteria to specific diseases, leading to a broadening of his ideas, which eventually made its way into the general medical and dental curricula. Prior to the new research that is emerging, few dentists would argue with the need to 'kill germs on contact' in order to deal with the more common oral degenerative conditions, such as gum disease and tooth decay. Generations of oral care products, aimed at both the general public and dental profession, have made their way into our society-products that advertise their superiority in eradicating bacteria inhabiting the mouth. Thankfully, this approach never succeeded. According to some experts, and based on the emerging science, this could have unleashed an "ecological Armageddon". In the same way that antibiotic overuse disrupts the helpful, friendly bacteria in the gut, a similar scenario occurs when we try to disinfect, or worse sterilize, the oral cavity [7].

Pioneering research performed by both Dr. P.D. Marsh of the Leeds Dental Institute in the U.K., and Dr. D. Relman of Stanford University points to a new paradigm in our understanding of oral degenerative conditions. Their research depicts the mouth as an ecosystem-a network or community of microorganisms that live in cooperation with one another and, most importantly, live in 
symbiosis with us, their hosts. Although this discovery comes as no surprise to any biologist, it is a groundbreaking understanding for dental clinicians [8]. For generations, the universal belief was "plaque is bad, get rid of it." The thought of the oral biofilm plaque as something that could be helpful and friendly, let alone essential to one's overall health, is a direct contradiction of everything previously accepted by dental institutions [9].

These studies have illuminated a new scientific understanding, revealing the fact that the state of the mouth is far from uniform in its distribution of bacterial species. Planktonic organisms, the organisms that populate the freely flowing saliva, profoundly differ from the organisms that adhere to the surfaces of the teeth, gums, and mucous membranes. Organisms that exist solely in this adherent layer of biofilm represent over $99.9 \%$ of all bacteria in the mouth, and exist in highly organized communities, which we refer to as the oral microbiome. They create internal architecture to optimize nutrient absorption, waste removal and overall survival. They also communicate within species to enable them to sense population densities and adjust their growth rates. Planktonic organisms even communicate between species through a process called quorum sensing, [10] using both chemical markers as well as shared genetic information in an effort to ensure the community, or ecosystem, survives and thrives. What is particularly interesting about this oral microbiome is that every species on the planet with a mouth has and will always have an oral ecology or ecosystem, unique to that species [11]. Furthermore, according to Darwin's Law of Natural Selection, each of these oral ecosystems has contributed to the survival and longevity of their hosts. If any of these environments become pathological to their hosts, the resident organisms would themselves have less of a chance to thrive. Therefore, the oral microbiome is a vital, natural and supportive component to oral health and protection of our teeth, gums and mouth linings. The problem then, is not that the oral microbiome exists, but rather it can become unbalanced and unhealthy. Disease occurs when the oral microbiome loses homeostasis, or balance.

The unhealthy or imbalanced oral microbiome is analogous to a garden that has become overgrown by weeds. One approach to this problem would be to "bulldoze" the garden down to the soil. However, we know that when there is bare soil, the very first kinds of plants that take root are the weeds. This illustrates the past approach in oral care therapeutics, which attempted to strip away the oral microbiome, using any kind of detergent or emulsifierthe kinds normally found in conventional toothpastes. By doing so, we are creating an opening in an ecological niche, where "stripped bare surfaces" become recolonized quickly, often by more virulent microorganisms (i.e., the weeds), rather than the friendlier, helpful, odorless bacteria that take more time to develop. Even here, emerging science is teaching us another new understanding: the virulent microorganisms are oftentimes the same "friendly" organisms that, in a balanced state, are helpful and cooperative (i.e., benign and beneficial). In the absence of homeostasis, they transform themselves into a pathogenic state through a process known as pleomorphism. In microbiology, pleomorphism is the ability of bacteria to also alter their shape or size in response to adverse environmental conditions [12]. A second approach to an imbalanced oral microbiome or "garden of weeds" would be the introduction of antimicrobials, analogous to the "pesticide approach" to apply "weed killers." Once again, this presents an even bigger problem. In this circumstance, we begin Darwin's process of selective growth of resistant organisms with consequences that are well understood to hospital-based infectious disease specialists who encounter the threat of microbial resistance with patient's every day. In the mouth, antimicrobial use results in a destruction of the beneficial flora that protects and heals teeth, gums, and mucus membranes. Based on this emerging science of the oral microbiome, a more enlightened approach treats this ecosystem as an organic garden [13]. In this garden, there are no "bulldozers" and no "weed killers." Rather, appropriate nutrients and microminerals to "feed the soil and keep the garden healthy." By choosing selected nutrients and trace minerals to promote homeostasis, the health of beneficial microorganisms is supported in this essential ecosystem. In return, the presence of pathogenic, planktonic organisms, or analogously, "weeds" which never get a chance to grow. To state it more simply, the application of nutrientdense "food" to an unbalanced biofilm, helps the "good guys" get healthier "crowding out the weeds" on their own, thus restoring an essential balance to a very important community for vibrant oral health and wellness.

CS Bioscience, a company that has been a leader in research and development in the application of the new emerging science of the oral microbiome in oral care therapeutics, has developed a proprietary and all-natural bioeffective dental formulation comprised of key nutritional components, homeopathic microminerals, and herbal ingredients [14]. This unique approach has demonstrated very strong efficacy in comparison to competitive oral care products based on previous scientific beliefs (i.e. "killing plaque"). An initial product being introduced, a toothpaste substitute claims to nourish the helpful, friendly, odorless bacteria that constitute a balanced biofilm, keeping it from degenerating into an unbalanced state, causing the oral microbiome to lead to oral breakdown. Pilot clinical research has demonstrated that this formulation promoted a "clear, thin, slippery" biofilm, a sign of a healthy oral microbiome [15]. Multiple study participants reported that their teeth felt "smooth and slick hours after brushing, even overnight." Other users claimed their "teeth still felt clean in the morning" after using the product for several days. Where gums are robustly resistant to the challenges that lead to puffiness and bleeding, this "nourishing" approach in oral care therapeutics supports the oral environment's natural ecology back into balance promoting healthier gums [16]. Another benefit has also been this particular formulation's ability shown to relieve conditions for those who suffer from xerostomia and oral mucositis commonly experienced during cancer treatment such as radiation therapy and chemotherapy. Dry conditions and burning mouths are also pathways that lead to tooth decay. This new frontier of oral care products that addresses the new understanding of the oral microbiome is just beginning to be explored. Therapeutic results never demonstrated by previous approaches in by oral care products 
has opened up the prospect of reversing the epidemic incidence of oral disease, specifically periodontal disease. The links of oral disease to many debilitating systemic illnesses, including fatal coronary heart and cerebrovascular diseases, [17] premature low weight birth and pre-eclampsia during pregnancy, $[18,19]$ respiratory disease, Alzheimer's disease and even cancer [20] has profound implications for healthcare. The strong correlation between oral disease and systemic wellness also has enormous implications in reducing healthcare costs. A 2006 study by Aetna and Columbia University's College of Dental Medicine on 145,000 patients found up to a $21 \%$ reduction in healthcare costs associated with the treatment of major systemic diseases, cardiovascular disease, cerebrovascular disease and diabetes, when oral health is improved [20]. A similar study repeated in Japan produced a nearly identical statistic. The healthcare costs in the United States related to these systemic conditions are over three quarters of a trillion dollars annually placing improvement of the nation's oral health on the "front line" of future healthcare strategies.

\section{References}

1. U.S. Department of Health and Human Services. Oral Health in America: A Report of the Surgeon General. Rockville, MD: U.S. Department of Health and Human Services, National Institute of Dental and Craniofacial Research, National Institutes of Health, 2000. p. 286.

2. Lederberg, J., \& Mccray, A. T. (2001). 'Ome Sweet 'Omics-A Genealogical Treasury of Words. The Scientist 15, 8-10.

3. Dewhirst, F. E., Chen, T., Izard, J., Paster, B. J., Tanner, A. C., Yu, W. H., et al. (2010). The human oral microbiome. J Bacteriol, 192, 5002-17.

4. Leivadaros, E., van der Velden, U., Bizzarro, S., Ten Heggeler, J. M., Gerdes, V. E., Hoek, F. J., et al., (2005). A Pilot Study into measurements of markers of atherosclerosis in periodontitis. J Periodontol, 76(1) 121-8.

5. Dortbudak, O., Eberhardt, R., Ulm, M. \& Persson, G. R. (2005). Periodontitis, a Marker of Risk in Pregnancy for Preterm Birth. J Clin Periodontol, 32(1), 45-52

6. Van, Duynhoven, J., Vaughan, E., Jacobs, D., Kemperman, R., Velzen, E. Van., Gross, G., et.al., (2011). Metabolic fate of polyphenols in the human superorganism. Proc. Natl. Acad. Sci USA, 108, 4531-4538.

7. Brading, M. G. \& Marsh, P. D. (2003). The oral environment: the challenge for antimicrobials in oral care products. Int Dent J. 53(6), 353-62.

8. Marsh, P. D. (2003). Are dental diseases examples of ecological catastrophes? Microbiology, 149, 279-94.

9. Fejerskov, 0. (2004). Changing paradigms in concepts on dental caries: consequences for oral health care. Caries Res, 38(3), 182-91.
10. Miller, M. B., \& Bassler, B. L. (2001). Quorum sensing in bacteria. Annu Rev Microbiol. 55, 165-99.

11. Dethlefsen, L., McFall-Ngai, M., \& Relman, D. A. (2007). An ecological and evolutionary perspective on human-microbe mutualism and disease. Nature. 449(7164), 811-8.

12. Joshi, H. M., \& Toleti, R. S. (2009). Nutrition induced pleomorphism and budding mode of reproduction in Deinococcus radiodurans. BMC Research Notes, 2(123).

13. Fejerskov, O. (2004). Changing paradigms in concepts on dental caries: consequences for oral health care. Caries Res, 38(3), 182-91.

14.DISCLAIMER: The author would like to disclose his position as cofounder/chairman of CS Bioscience, an oral care biosciences company that has performed more than 16 years of research and development in the oral microbiome science arena. The opinions or views expressed in this journal article are those of the authors and do not necessarily reflect the opinions and recommendations of the publisher, journal, guest editors, or supporters.

15. Daniels., Anita. H., Jefferies., \& Steven, R. (2007). Analysis of Capacity of Novel, Antioxidant Toothpaste to Reduce Gingival Inflammation in Pilot, Small-population Clinical Study: Comparison to Levels of Gingival Inflammation Reduction Reported in Historical Control and Therapeutic Tooth brushing Studies. Temple University School of Dentistry, Clinical Research and Biomaterials Analysis and Review Report submitted to CS Bioscience, New York.

16. Pameijer, C. H., Grande, N., Plotino, G., Butti, A., Lerda, A., \& Pasquali, V. (2008). An Evaluation of the Effectiveness of an Experimental Oral Therapy Paste (RevitinTM with NuPath ${ }^{\circledR}$ Bioactives) on Oral Soft Tissue Health. Report on single blind clinical research study performed at Catholic University of Rome, Italy submitted to CS Bioscience, New York.

17. Morison, H. I., Ellison, L. F., \& Taylor, G. W. (1999). Periodontal disease and risk of fatal coronary heart and cerebrovascular diseases. $J$ Cardiovasc Risk, 6(1), 7-11.

18. Dasanayake, A. P. (1998). Poor periodontal health of the pregnant woman as a risk factor for low birth weight. Ann Periodontol, 3(1), 206-12.

19. Offenbacher, S. (1993). Periodontal pathogenic mechanisms of periodontitis-associated pregnancy complications. Annals Periodontology, 3, 233-250.

20. Michaud, D. (2007). Periodontitis and tooth loss and the subsequent risk of pancreatic cancer. J National Cancer Institute, 99, 171-175.

21. Report by Aetna/Columbia University College of Dental Medicine, March 20, 2006, BMC Health Serv Res. 2006; 6: 103. An examination of periodontal treatment and per member per month (PMPM) medical costs in an insured population. 